\title{
BMJ Open Protocol for a multicentre, parallel-arm, 12-month, randomised, controlled trial of arthroscopic surgery versus conservative care for femoroacetabular impingement syndrome (FASHIoN)
}

D R Griffin, ${ }^{1}$ E J Dickenson, ${ }^{2}$ P D H Wall, ${ }^{2} \mathrm{~J} \mathrm{~L} \mathrm{Donovan,}{ }^{3} \mathrm{~N}$ E Foster, ${ }^{4} \mathrm{C}$ E Hutchinson, ${ }^{2} \mathrm{~N}$ Parsons, ${ }^{2} \mathrm{~S}$ Petrou, ${ }^{2}$ A Realpe, ${ }^{2} \mathrm{~J}$ Achten, ${ }^{2} \mathrm{~F}$ Achana, ${ }^{2}$ A Adams, ${ }^{2} \mathrm{M} \mathrm{L}$ Costa, ${ }^{2} \mathrm{~J}$ Griffin, ${ }^{2} \mathrm{R}$ Hobson, ${ }^{2} \mathrm{~J}$ Smith, ${ }^{2}$ on behalf of the FASHIoN Study Group

To cite: Griffin DR, Dickenson EJ, Wall PDH, et al. Protocol for a multicentre, parallel-arm, 12-month, randomised, controlled trial of arthroscopic surgery versus conservative care for femoroacetabular impingement syndrome (FASHION). BMJ Open 2016;6:e012453. doi:10.1136/bmjopen-2016012453

- Prepublication history and additional material is available. To view please visit the journal (http://dx.doi.org/ 10.1136/bmjopen-2016012453).

Received 29 April 2016 Revised 23 May 2016 Accepted 16 June 2016

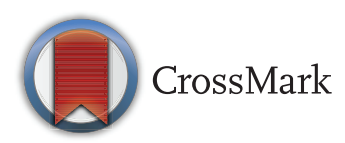

For numbered affiliations see end of article.

Correspondence to Prof D R Griffin; damian.griffin@warwick.ac.uk

\section{ABSTRACT}

Introduction: Femoroacetabular impingement (FAI) syndrome is a recognised cause of young adult hip pain. There has been a large increase in the number of patients undergoing arthroscopic surgery for FAl; however, a recent Cochrane review highlighted that there are no randomised controlled trials (RCTs) evaluating treatment effectiveness. We aim to compare the clinical and cost-effectiveness of arthroscopic surgery versus best conservative care for patients with FAl syndrome.

Methods: We will conduct a multicentre, pragmatic, assessor-blinded, two parallel arm, RCT comparing arthroscopic surgery to physiotherapy-led best conservative care. 24 hospitals treating NHS patients will recruit 344 patients over a 26-month recruitment period. Symptomatic adults with radiographic signs of FAl morphology who are considered suitable for arthroscopic surgery by their surgeon will be eligible. Patients will be excluded if they have radiographic evidence of osteoarthritis, previous significant hip pathology or previous shape changing surgery. Participants will be allocated in a ratio of $1: 1$ to receive arthroscopic surgery or conservative care. Recruitment will be monitored and supported by qualitative intervention to optimise informed consent and recruitment. The primary outcome will be pain and function assessed by the international hip outcome tool 33 (iHOT-33) measured 1-year following randomisation. Secondary outcomes include general health (short form 12), quality of life (EQ5D$5 \mathrm{~L}$ ) and patient satisfaction. The primary analysis will compare change in pain and function (iHOT-33) at 12 months between the treatment groups, on an intention-to-treat basis, presented as the mean difference between the trial groups with $95 \% \mathrm{Cls}$. The study is funded by the Health Technology Assessment Programme (13/103/02).

Ethics and dissemination: Ethical approval is granted by the Edgbaston Research Ethics committee (14/WM/0124). The results will be disseminated

\section{Strengths and limitations of this study}

- This trial is multicentre, pragmatic and randomised, making results generalisable across the NHS.

- Further strengths include a large sample size and the robust procedures to assess treatment fidelity.

- The trial has a large sample size (344).

- There are robust procedures to assess treatment fidelity.

through open access peer-reviewed publications, including Health Technology Assessment, and presented at relevant conferences.

Trial registration number: ISRCTN64081839; Pre-results.

\section{BACKGROUND}

Until recently, there was little understanding of the causes of hip pain in young adults. Since first described in 2003, there has been increasing recognition of the syndrome of femoroacetabular impingement (FAI), which seems to account for a proportion of the previously undiagnosed cases of hip pain in young adults. $^{1}{ }^{2}$ Subtle deformities of hip shape combine to cause premature contact between the femoral neck and the acetabular rim which may result in hip pain. ${ }^{13}$ These shape abnormalities typically divide into three categories: ${ }^{34}$

- Cam-type, in which the femoral head is oval rather than round, or there is bony prominence on the femoral neck;

- Pincer-type, in which the rim of the acetabulum is excessively prominent, in one or more areas of its circumference; 
- Mixed-type hip impingement, a combination of cam and pincer types.

Surgery can be performed to reshape the bony contour of the proximal femur and/or acetabular rim in order to prevent impingement. Surgery for FAI has evolved more quickly than our understanding of the epidemiology or natural history of the condition, ${ }^{5}{ }^{6}$ and is becoming an established treatment for FAI. ${ }^{7}$ The risks of complications from open surgery are greater than those for arthroscopic surgery and current evidence suggests that the outcomes of arthroscopic treatment for the symptoms of FAI are comparable to open surgery. ${ }^{8} 9$ Consequently, hip arthroscopy for FAI is a rapidly growing new cost pressure for health providers. ${ }^{10}$ However, a recently published Cochrane review highlighted the absence of randomised controlled trials (RCTs) comparing FAI surgery with conservative care such as physiotherapist-led exercise. ${ }^{11}$

Physiotherapy has also been shown to be beneficial in patients with FAI syndrome. ${ }^{12}{ }^{13}$ During a successful feasibility study (HTA 10/41/02), a programme of physiotherapist-led conservative care was developed called personalised hip therapy (PHT). ${ }^{14}$

\section{Aims of trial}

We aim to compare the clinical and cost-effectiveness of arthroscopic surgery versus physiotherapist-led conservative care (PHT) in patients with symptoms of FAI syndrome.

\section{METHODS/DESIGN}

This trial will be conducted in accordance with the Medical Research Council's Good Clinical Practice principles and guidelines, the Declaration of Helsinki, Warwick Clinical Trials Unit (WCTU) standard operating procedures $(\mathrm{SOPs})$, relevant UK legislation and the trial protocol. Ethical approval was granted on 1 May 2014 (14/WM/0124), by the Edgbaston Research Ethics committee (current approved protocol V.3.1 20/01/ 2016). The trial will be reported in line with the CONSORT statement. This full trial follows a successful feasibility and pilot trial (HTA10/41/02). ${ }^{14}$

\section{Trial design and setting}

This is a protocol for the full UK RCT of arthroscopic surgery for hip impingement versus best conservative care (FASHIoN). We will conduct a multicentre, pragmatic, assessor-blinded, parallel arm, 12 months, 1:1 RCT of hip arthroscopy versus conservative care for FAI assessing patient pain, function, general health, quality of life, satisfaction and cost-effectiveness. There is an integrated qualitative recruitment intervention that includes interviews with recruiters and patients, and observations of recruitment appointments to ensure patients have the opportunity to fully consider participation in the trial. ${ }^{15}$

We hypothesise that arthroscopic surgery is superior to conservative care at 12 months for self-reported hip pain and function for patients with FAI syndrome. The trial will be conducted on consenting patients treated in the NHS. Hospitals participating in FASHIoN will have an organised hip arthroscopy service treating at least 20 patients with arthroscopic surgery for FAI per year.

\section{Target population}

We intend to recruit a cohort of typical patients with FAI deemed suitable for arthroscopic surgery. This included patients who may have already received a course of physiotherapy.

\section{Inclusion criteria}

- Age $\geq 16$ (no upper age limit);

- Symptoms of hip pain-patients may also have symptoms of clicking, catching or giving way;

- Radiographic evidence of pincer- and/or cam-type FAI morphology on plain radiographs and crosssectional imaging, defined as:

- Cam morphology—an $\alpha$ angle $>55^{\circ} ;{ }^{16}$

- Pincer morphology - a lateral centre edge angle of $>40^{\circ}$ or a crossover sign on the anteroposterior radiograph of the pelvis; ${ }^{17}$

The treating surgeon believes the patient would benefit from arthroscopic FAI surgery;

The patient is able to give written informed consent and to participate fully in the interventions and follow-up procedures.

\section{Exclusion criteria}

- Evidence of pre-existing osteoarthritis, defined as Tonnis grade $>1,{ }^{18}$ or more than $2 \mathrm{~mm}$ loss of superior joint space width on anterio-posterior pelvic radiograph; ${ }^{19}$

- Previous significant hip pathology such as Perthes' disease, slipped upper femoral epiphysis or avascular necrosis;

- Previous hip injury such as acetabular fracture, hip dislocation or femoral neck fracture;

- Previous shape changing surgery (open or arthroscopic) in the hip being considered for treatment.

\section{Participant identification, invitation, recruitment and baseline data collection}

Patients who complain of hip pain, who do not already have a diagnosis of hip osteoarthritis, will be identified as potential participants by screening referral letters to collaborating surgeons. Research nurses/associates will keep accurate screening logs to identify if these potential participants meet the eligibility criteria. Once diagnosed with FAI syndrome by the surgeon, and deemed eligible for the trial, the patient will be given a trial information sheet (see online supplementary files 1 and 2) and referred to a trained recruiter for a trial information consultation. During this consultation, patients can discuss the trial, participation will be offered and informed consent obtained (see online supplementary files 3 and 4). It will be explained that participation is voluntary and patients can withdraw at any time. Once 
consent is obtained, and prior to treatment allocation, baseline patient reported outcomes will be collected (see the Outcome measures section).

In order to optimise recruitment and informed consent, trained qualitative researchers will observe recordings of the surgeons' and research associate/ nurses' trial information consultations (see online supplementary files 2 and 4), to identify communication patterns that facilitate or hinder patient recruitment ${ }^{15}$ (see figure 1). In-depth interview with the recruiters will be undertaken to identify clear obstacles and hidden challenges to recruitment, including the influence of patient preferences and equipoise. ${ }^{20}$ Research teams will be interviewed to identify clinician equipoise, patient pathway from eligibility to consent and staff training needs at each participating site. ${ }^{15}$ Findings will be fed back to the CI and trial management group, so that practice can be reviewed and any necessary changes (including additional training) implemented. The number of eligible patients, the percentages of these that are approached and consented to be randomised will be monitored at each site.

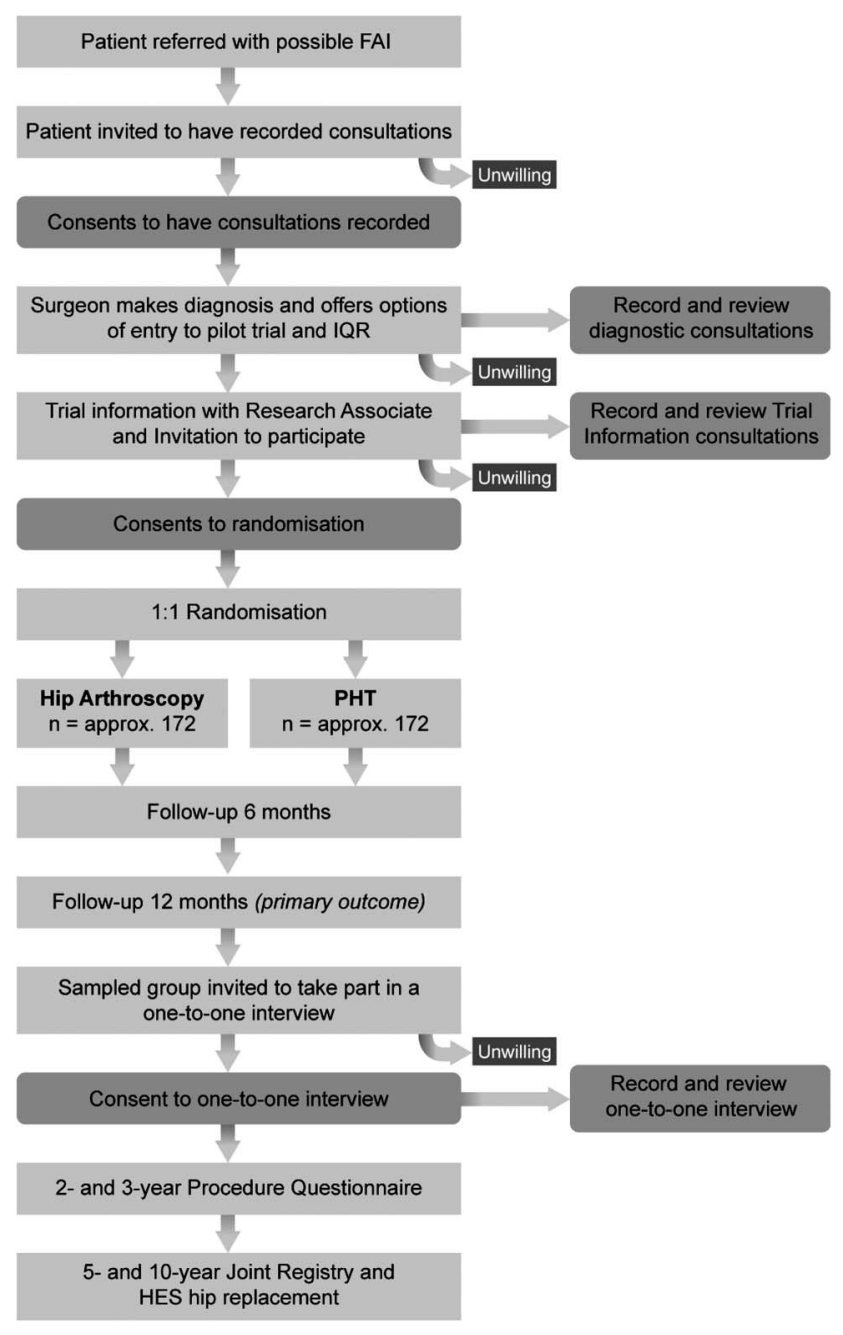

Figure 1 Participant flow diagram.
This research will be linked, through Donovan, to the Quintet programme of research within the MRC ConDuCT-II (Bristol) Trial Methodology Hub.

\section{Randomisation}

Participants will be randomised, in a 1:1 ratio, to arthroscopic surgery or PHT using a computer-generated sequence. Allocation will be made by the research nurse/associate via a centralised telephone randomisation service provided remotely by WCTU. Allocation concealment will be ensured, as the randomisation programme will not release the randomisation code until the patient has been recruited into the trial. In order to improve baseline balance between intervention group samples, a minimisation (adaptive stratified sampling) algorithm will be implemented using study site and impingement type (cam, pincer or mixed) factors. Research nurses/associates who recruit participants will ensure they are referred for the allocated intervention. Patients and clinicians cannot be blind to treatment allocation. However, outcome assessors will be blind to the treatment delivered.

\section{Interventions}

The two interventions will start as soon as possible after randomisation. We will record dates of randomisation and the start of allocated treatment. As this is a pragmatic trial, participants were not prohibited from undergoing any additional/concomitant care.

\section{Arthroscopic surgery}

Arthroscopic surgery will be completed by a Consultant Surgeon delivering hip arthroscopy as part of their routine practice. Arthroscopic hip surgery will be performed under general anaesthesia according to the surgeon's usual practice. Shape abnormalities and consequent labral and cartilage pathology will be treated. Bony resection at the acetabular rim and at the head-neck junction will be assessed by intraoperative image intensifier radiographs and/or satisfactory impingement-free range of movement of the hip. Patients will be allowed home when they can walk safely with crutches (usually within 24 hours). On discharge, patients will be referred for a course of rehabilitation as per usual care for that surgeon. We will not specify a protocol for this postoperative physiotherapy, but will record the surgeons' routine postop care and any case-by-case changes to this. Care will be taken to ensure that physiotherapists delivering postoperative care to FASHIoN trial participants are different from those trained and providing PHT in order to avoid contamination between groups. Patients will also have a postoperative MRI after 6 weeks.

In order to ensure the fidelity of the surgery and to identify participants for a secondary analysis, a panel of international experts will review operation notes, intraoperative images and postoperative MRI scans to assess whether adequate surgery was undertaken. This panel 
includes: Mark Philippon (USA), Martin Beck (Switzerland), John O'Donnell (Australia) and Professor CEH (UK).

\section{Personalised hip therapy}

PHT is a package of physiotherapist-led best conservative care for FAI. It was developed during the feasibility study and 'road-tested' during the pilot trial (HTA 10/41/ 02). ${ }^{14}$ The care being offered represents a consensus of what physiotherapists, physicians and surgeons regard as 'best conservative care' for FAI. PHT will be delivered by a senior physiotherapist at each site, who will be trained at a FASHIoN PHT workshop, and supported in PHT delivery by a physiotherapy research facilitator.

PHT consists of four key components:

1. An assessment of pain, function and range of hip motion,

2. Patient education and advice,

3. Help with pain relief (which may include up to one radiographic-guided intra-articular steroid injection where pain prevents performance of the exercise programme),

4. An exercise programme that has the key features of individualisation, progression and supervision.

The intervention is delivered over a minimum of six patient contacts (at least three of which must be face-to-face treatment contacts, others can be by telephone and email) over a period of 6 months. In situations where the patient needs additional review, support or guidance, further sessions with the physiotherapist are permitted up to a maximum of 10 contacts. Evidence of exercise individualisation, supervision and progression will be sought from individual participant physiotherapy case report forms (CRFs). Accuracy of CRFs will be audited against the physiotherapist's treatment notes.

The PHT CRFs will be assessed for intervention fidelity to identify participants for a secondary analysis by the panel that developed the protocol for PHT, including: Professor NEF (Senior Academic Research Physiotherapist), Ivor Hughes and David Robinson (UK; Extended Scope Musculoskeletal Physiotherapists) and PW (Academic Orthopaedic Surgeon).

Crossover of participants between interventions can be problematic in trials of this nature. In order to minimise, this care will be taken prior to enrolment in the trial to ensure potential participants:

- Are willing to receive either intervention,

- Understand both treatments are thought to provide benefit,

- Are willing to remain with their allocation for 12 months,

- Understand that both interventions may take 6 months to improve symptoms. ${ }^{12} 19$

In instances where patients are not satisfied with how their treatment is progressing prior to reaching the primary outcome, they will be able to have a further consultation with their treating surgeon where they would be treated in their best interests.
Risks and benefits

Both interventions are thought to provide benefit in patients with FAI. The short-term risks of this study relate to the two interventions. These risks are described below and inform the expected serious adverse events (SAEs).

Hip arthroscopy requires a general anaesthetic. The risk of complications from hip arthroscopy is about $1-2 \%$. These include:

- Infection-thought to be $<1$ in 1000 .

- Bleeding-possibly causing bruising or a local haematoma.

- Traction-related-in order to perform hip arthroscopy, traction is required to separate the hip joint surfaces. Sometimes after the procedure, the pressure from the traction can cause some numbness in the leg. The numbness usually resolves within a few hours or days.

- Osteonecrosis-during surgery, the blood supply to the hip joint could be damaged. However, there are no reported cases of osteonecrosis following arthroscopic FAI surgery.

- Femoral neck fractures-this is also a very rare complication. This complication would require a further procedure to fix the fracture.

\section{Personalised hip therapy}

There are some small risks with pain medications and joint injection. However, the main risk is muscle soreness and transient increases in pain from the exercises that will be undertaken.

\section{Outcome measures}

Baseline data will be collected from participants once consent is obtained and prior to randomisation.

\begin{tabular}{|c|c|}
\hline Time point & Data collection \\
\hline Baseline & $\begin{array}{l}\text { Demographics, physical activity } \\
\text { (UCLA Activity Scale), }{ }^{29} \text { iHOT-33, } \\
\text { SF-12,EQ-5D } \\
\text { Preoperative imaging, economics } \\
\text { questionnaire }\end{array}$ \\
\hline Intervention & $\begin{array}{l}\text { Operation notes and photographs; } \\
\text { or PHT log. Complications records } \\
6 \text { weeks post start of intervention. } \\
\text { Postoperative MRI (surgery } \\
\text { intervention only) }\end{array}$ \\
\hline 6 months & $\begin{array}{l}\text { iHOT-33, SF-12, EQ-5D, resource } \\
\text { usage, adverse events }\end{array}$ \\
\hline $\begin{array}{l}12 \text { months (primary } \\
\text { outcome) }\end{array}$ & $\begin{array}{l}\text { iHOT-33, SF-12, EQ-5D, patient } \\
\text { satisfaction, resource usage, } \\
\text { adverse events }\end{array}$ \\
\hline 2 years & Further procedures questionnaire \\
\hline 3 years & Further procedures questionnaire \\
\hline 5 and 10 years & $\begin{array}{l}\text { Linkage to National Joint Registry } \\
\text { and HES to identify need for hip } \\
\text { replacement }\end{array}$ \\
\hline
\end{tabular}


Follow-up questionnaires will be administered centrally by a data clerk via post. If participants fail to respond, they will be contacted via telephone, email or via their next of kin where necessary. Table 1 lists the data collected and at which follow-up time points.

\section{Primary outcome}

The primary outcome measure is hip pain, function and hip-related quality of life using the International Hip Outcome Tool-33 (iHOT-33) at 12 months following randomisation. iHOT-33 is a validated hip-specific patientreported outcome tool which measures health-related quality of life in young, active patients with hip disorders. ${ }^{21}$ It consists of the following domains: symptoms and functional limitations, sports and recreational activities, job-related concerns and social, emotional and lifestyle concerns.

We chose it following our feasibility and pilot study as:

- It is more sensitive to change than other hip outcome tools, ${ }^{21}$

- It does not show evidence of floor or ceiling effects in patients undergoing hip arthroscopy, ${ }^{21}$

- Patients were involved extensively in item generation; so we can be confident that it measures what is most important to patients, ${ }^{21}$

- There is an independently determined minimally clinically important difference (MCID), ${ }^{21}$

- It is used as the principal outcome measure for the UK Non-Arthritic Hip Registry; mandated for arthroscopic FAI surgery by the National Institute of Health and Care Excellence (NICE). ${ }^{10}$

\section{Secondary outcome measures}

Health-related quality of life: EQ-5D 5L

This is a validated measure of health-related quality of life, consisting of a five-dimensional health status classification system and a separate visual analogue scale. EQ-5D is applicable to a wide range of health conditions and treatments and provides a simple descriptive profile and a single index value for health status. ${ }^{22}$ Responses will be converted into health utility scores using established algorithms. ${ }^{23}$

\section{General health: Short Form-12 Health Survey V.2}

This is a validated and widely used health-related quality-of-life measure, particularly including hip conditions and treatments. ${ }^{24}$ SF-12 is able to produce the physical and mental component scales originally developed from the SF-36 with considerable accuracy but with far less respondent burden. ${ }^{25}$ Responses will be converted into health utility scores using established algorithms. $^{26}$

\section{Patient satisfaction}

Using questions that our team (Foster) has used in previous trials with musculoskeletal pain patients, ${ }^{27}$ we will measure two distinct dimensions of satisfaction in all participants during follow-up: 'Overall, how satisfied are you with the treatment you received?' and 'Overall, how satisfied are you with the results of your treatment?' Responses are on a 5-point Likert scale. These questions are in line with previous studies of patient satisfaction which show that the majority of patients express overall satisfaction with the care they received, but fewer express overall satisfaction with the clinical outcomes resulting from their care.

\section{Qualitative assessment of outcome}

We will conduct in-depth interviews one-to-one with a purposively selected sample of 25-30 participants in each of the trial groups, including older and younger, male and female, more and less active and more and less satisfied participants recruited at different trial sites. The qualitative interviews will supplement the quantitative outcomes. Interviews will explore experiences of the trial processes, the treatments and the consequences of treatment to participants' lives, health and well-being.

\section{Adverse events}

We will record the number and type of adverse events (AEs) up to 12 months. Any AEs will be reported on the appropriate CRF and returned to WCTU. Any SAEs will be faxed to WCTU, within 24 hours of the local investigator becoming aware, where the Chief Investigator will determine causality and expectedness. SAEs deemed unexpected and related to the trial will be reported to the research ethics committee within 15 days.

\section{Resource usage}

Information on healthcare resource use will be collected by incorporating questions within the patient follow-up questionnaires. We confirmed the feasibility and acceptability of this approach in our pilot trial, and patient self-reported information on service use has been shown to be accurate in terms of the intensity of use of different services. ${ }^{28}$

\section{Need for further procedures}

We will record any further treatments performed in both groups, such as hip arthroscopy, open hip preservation surgery, hip replacement or additional 'out of trial' physiotherapy. We propose to ascertain the need for further procedures by questionnaire at 2 and 3 years. We also propose a 5 and 10-year no-cost ascertainment of hip replacement by linkage to the UK National Joint Registry (NJR) and Hospital Episode Statistic (HES) databases.

\section{Sample size calculation}

The development work for iHOT-33 reported a mean iHOT-33 score of 66 and an SD of 19.3 in a heterogeneous population with a variety of hip pathologies. The baseline iHOT-33 data from our pilot trial (HTA grant 10/41/02) suggests the target population of patients being considered for hip arthroscopy for FAI have lower scores with less variability, with a mean of 33 and SD of 16. The MCID for iHOT-33 in this population is 6.1 points. $^{21}$ 
Our sample size calculation is therefore based on an SD of 16 and a between-group MCID of 6.1: a standardised effect difference between groups at 12 months of 0.38 . The expected sample size for $90 \%$ power to detect an effect size of 0.38 at 12 months, at a $5 \%$ significance level, assuming an approximately normal distribution of the iHOT-33 score is 292. Allowing for $15 \%$ loss to follow-up at 12 months, we will recruit a sample of 344 participants over 26months in the UK (172 in each group).

\section{Statistical analysis}

The primary analysis will be of differences in hip-related quality of life (iHOT-33) at 12 months between the two treatment groups, blinded, on an intention-to-treat basis and presented as the mean difference between the trial groups with a 95\% CI. iHOT-33 data will be assumed to be normally distributed; possibly after appropriate variance-stabilising transformation.

The minimisation randomisation procedure should ensure treatment group balance across recruiting sites. We have no reason to expect that clustering effects will be important for this study, but the possibility of such effects will be explored as part of the analysis. ${ }^{33}$ We plan to account for clustering by generalising a conventional linear (fixed-effects) regression approach to a mixed-effects modelling approach; where patients are naturally grouped by recruiting sites (random-effects) and, if amenable to analysis, also by physiotherapist and surgeon. This model will formally incorporate terms that allow for possible heterogeneity in responses for patients due to the recruiting centre, in addition to the fixed effects of the treatment groups, and patient characteristics that may prove to be important moderators of treatment effect such as age, gender and FAI type. This analysis will be conducted using specialist mixed-effects modelling functions available in the software packages Stata (StataCorp. 2015. Stata Statistical Software: Release 14. College Station, Texas, USA: StataCorp LP) and R (http://www.r-project.org/). All tests will be two-sided and considered to provide evidence for a statistically significant difference if $\mathrm{p}$ values are $<0.05$ (5\% significance level).

Secondary analyses will be performed using the above strategy for other approximately normally distributed outcome measures, including iHOT-33 at 6 months, SF-12 (and computed subscales) and EQ5D. Differences in dichotomous outcome variables such as AEs, complications related to the trial interventions and the need for further procedures will compared between groups using $\chi^{2}$ tests (or Fisher's exact test) and mixed-effects logistic regression analysis will be undertaken, adjusting for the stratifying variables, with differences between trial intervention groups quantified as ORs (and 95\% CIs). The temporal patterns of any AEs will be presented graphically and if appropriate, a time-to-event analysis (Kaplan-Meier survival analysis) will be used to assess the overall risk and risk within individual classes of AEs.
Ordinal scores for patient satisfaction will be compared between intervention groups using proportional odds logistic regression analysis, assuming that the estimated intervention effect between any pair of categories is equivalent.

Our inferences will be drawn from the intentionto-treat analysis. We will perform two exploratory secondary analyses. One will compare patients who received surgery and those who received conservative care. A second exploratory analysis will compare patients randomised to surgery or PHT and received treatment deemed to be of a high fidelity by the respective review panels. We plan to perform a subgroup analysis by FAI type because it is possible that treatment effect is moderated by type. We anticipate that adequate steps have been taken to prevent crossovers from being a major issue for this study. Therefore, we expect the main intention-to-treat analysis to provide definitive results. An independent Data Monitoring Committee (DMC) will monitor crossovers and adherence to treatment and advise on appropriate modifications to the statistical analysis plan as the full trial progresses.

The initial feasibility and pilot studies (HTA 10/41/ 02) were designed explicitly to assess feasibility and measure recruitment rates, and not to estimate treatment effectiveness. Data from the pilot will be pooled with data from the full trial, and analysed together.

\section{Economic analysis}

An economic evaluation will be integrated into the trial design and will be conducted from the recommended NHS and personal social services perspective. ${ }^{30}$ Cost-effectiveness will be calculated using both within trial and lifetime horizons. Data will be collected on the health and social service resources used in the treatment of each trial participant until 12 months.

An incremental cost-effectiveness analysis, expressed in terms of incremental cost per quality-adjusted life year gained, will be performed. Results will be presented using incremental cost-effectiveness ratios and costeffectiveness acceptability curves generated via nonparametric bootstrapping.

\section{Qualitative interview analysis}

Participant interview transcripts will be analysed thematically, using methods of constant comparison derived from grounded theory. ${ }^{31}$ Emerging themes will be explored, looking for shared or disparate views among patients about their experiences, and among clinicians about their experiences of delivering the trial interventions. Focused conversation analysis will be undertaken on sections of recruitment appointments, and compared with the six-step good recruitment model developed in the pilot study to identify aspects of RCT presentation that are unclear, disrupted or hinder recruitment. ${ }^{152032}$ 


\section{Data management}

All of the data collected in this trial will be entered into a secure trial database held at WCTU. All data collected will be anonymised after the collection of baseline demographic data, and all participants given a unique trial number. Identifiable participant data will be held in a locked filing cabinet and coded with a trial participant number to tag identifiable data to the outcome data. The WCTU quality assurance manager will undertake audits of trial records in accordance with WCTU SOPs.

A DMC will be established comprised of members who are independent of the sponsor and who do not have competing interests. The DMC will review trial progress, interim data and safety aspects of the trial. They will also review the statistical analysis plan. Any recommendations will be fed back to the trial steering committee (TSC) by the DMC chair. Outcomes will not be analysed until all primary outcome data are collected. The trial may be stopped prematurely if mandated by the research ethics committee, the DMC or if funding ceases.

\section{DISCUSSION}

This protocol paper describes the FASHIoN trial; a multicentre RCT comparing hip arthroscopy to best conservative care (PHT) in order to establish the most clinically and cost-effective treatment for patients with FAI syndrome. Further details of the trial protocol can be found on the ISRCTN registry (ISRCTN64081839). This protocol will also be used for a randomised trial in Australia (ACTRN12615001177549). The results of the trial will be disseminated at international meetings and in peer-reviewed journals; to participants via post and to the public via the trial website.

The main strengths of this trial are that it is multicentre, pragmatic and randomised, making results generalisable across the NHS. Further strengths include a large sample size and the robust procedures to assess treatment fidelity.

\section{Author affiliations \\ ${ }^{1}$ University of Warwick, University Hospitals of Coventry and Warwickshire NHS Trust, Coventry, UK \\ ${ }^{2}$ Warwick Medical School, University of Warwick, Coventry, UK \\ ${ }^{3}$ University of Bristol, University Hospitals Bristol NHS Foundation Trust, Bristol, UK \\ ${ }^{4}$ Arthritis Research UK Primary Care Centre, Research Institute of Primary Care and Health Sciences NIHR, Keele University, Keele, UK}

Twitter Follow Damian Griffin at @DamianGriffin

Collaborators The UK Comprehensive Research Network supported this trial. The FASHIoN Study Group: FASHIoN Surgeons: E Bache (The Royal Orthopaedic Hospital NHS Foundation Trust), M Bankes (Guys and St Thomas Hospital NHS Foundation Trust), G Bartlett (Royal Cornwall Hospitals NHS Trust), T Board (Wrightington, Wigan and Leigh NHS Foundation Trust), M Cronin (University Hospitals Coventry and Warwickshire NHS Trust), W Dandacholi (University College London Hospitals NHS Trust), S Eastaugh-Waring (North Bristol NHS Trust), M Fehily (Spire Manchester Hospital), E Fern (Ramsay Duchy Hospital), R Field (Southwest London Elective Orthopaedic Centre), M George (Guys and St Thomas Hospital NHS Foundation Trust), A Hashimi-Nejad (Royal National Orthopaedic Hospital NHS Trust),V Kavathapu (King's College Hospital NHS Foundation Trust), T Khan (Royal National Orthopaedic Hospital NHS Trist), N Kiely (Robert Jones and
Agnes Hunt Orthopaedic and District Hospital), P Latimer (Yeovil District Hospital NHS Trust), S Madan (Doncaster and Bassetlaw Hospitals NHS Foundation Trust), A Malviya (Northumbria NHS Trust), C Mcbryde (The Royal Orthopaedic Hospital NHS Foundation Trust), A Mohammed (Wrightington, Wigan and Leigh NHS Foundation Trust), M Norton (Royal Cornwall Hospitals NHS Trust), S Patil (NHS Greater Glasgow and Clyde), A Politis (The Royal Orthopaedic Hospital NHS Foundation Trust), A Rajpura (Wrightington, Wigan and Leigh NHS Foundation Trust), M Ramachandran (Bart's Health NHS Trust), G Stafford (Southwest London Elective Orthopaedic Centre), $S$ Sturridge (Frimley Health NHS Foundation Trust), P Thomas (Cardiff and the Vale University Health Board), C White (South Tees Hospitals NHS Foundation Trust), M Wilson (Royal Devon and Exeter NHS Trust), JP Whitaker (Robert Jones and Agnes Hunt Orthopaedic and District Hospital and Wrexham Maelor Hospital), M Williams (Plymouth Hospitals NHS Trust), J Witt (University College London Hospitals NHS Trust).

\section{FASHIoN Physiotherapists:}

E Jones (Yeovil District Hospital), S Baker (Yeovil District Hospital), J Stanton (Yeovil District Hospital), C Nicholls (Yeovil District Hospital), A Smeatham (Royal Devon \& Exeter NHS Foundation Trust), L Gosling (The Royal Orthopaedic Hospital NHS Foundation Trust), G Dickinson (Frimley Park Hospital), K Boulton (Frimley Health NHS Trust), J Goss (Epsom and St Hellier NHS Trust), R Venter (Guys and St Thomas Hospital NHS Foundation Trust), A Bennett (Bart's Health NHS Trust), R Simmons (University College London Hospitals NHS Trust), K Poll (University College London Hospitals NHS Trust), T Bergmann (University College London Hospitals NHS Trust), M Pilkington (Wrightington, Wigan and Leigh NHS Foundation Trust), $\mathrm{J}$ Armstrong (Wrightington, Wigan and Leigh NHS Foundation Trust), D Wright (Wrightington, Wigan and Leigh NHS Foundation Trust), P Dolphin (James Cook University Hospital), K Bainbridge (James Cook University Hospital), M Callum (Wansbeck General Hospital), S Lewis (Wansbeck General Hospital), E Smith (Wansbeck General Hospital), V Cornes (University Hospitals Coventry and Warwickshire), I Hughes (University Hospitals Coventry and Warwickshire), J Benfield (Royal National Orthopaedic Hospital), K Monnington (Royal National Orthopaedic Hospital), E Stewart (Royal National Orthopaedic Hospital), S Borrill (Doncaster Royal Infirmary), M Hyne (Robert Jones and Agnes Hunt Orthopaedic Hospital), N Harding (Robert Jones and Agnes Hunt Orthopaedic Hospital), S Dawson (Wrexham Maelor Hospital), M Willis (Wrexham Maelor Hospital), D Moore (Kings College Hospital), F Pressdee (The Royal Orthopaedic Hospital NHS Trust), K MacFarlane (The Royal Orthopaedic Hospital NHS Trust), A MacCauley (St Austell Community Hospital), D Cooke (Royal Cornwall Hospitals NHS Trust), B Fleck (University Hospitals Cardiff), P Dowrick (Derriford Hospital), J Ball (North Britsol NHS Trust), P Morrison (NHS Greater Glasgow and Clyde), C O'Donnell (NHS Greater Glasgow and Clyde), M Kennedy (Spire Manchester Hospital).

\section{FASHIoN Recruiting research nurses/research associates:}

S Turner (University Hospitals Coventry and Warwickshire), C Bryant (University Hospitals Coventry and Warwickshire), K Baddick (University Hospitals Coventry and Warwickshire), R McKeown (University Hospitals Coventry and Warwickshire), L Clarkson (University Hospitals Coventry and Warwickshire), A Lewis (Yeovil District Hospital), R Rowland-Axe (Yeovil District Hospital), A Grice (Royal Devon and Exeter NHS Trust), G Githens-Mazer (Royal Devon and Exeter NHS Trust), H Aughwan (The Royal Orthopaedic Hospital NHS Trust), F Moore (The Royal Orthopaedic Hospital NHS Trust), E Keeling (The Royal Orthopaedic Hospital NHS Trust), J Amero (Frimey Park NHS Foundation Trust), S Atkinson (Frimey Park NHS Foundation Trust), L Graves (Royal Cornwall Hospitals NHS Trust), E Fouracres (Royal Cornwall Hospitals NHS Trust), J Curtis (South West London Elective Orthopaedic Centre), T Kadiyirire (Guys and St Thomas Hospital NHS Foundation Trust), L Brackenridge (University College London Hospital), T Taylor (Wrightington, Wigan and Leigh NHS Foundation Trust), C Dobb (Northumbria NHS Trust), J Hinchliffe (Doncaster and Bassetlaw Hospitals NHS Foundation Trust), V Limbani (Royal National Orthopaedic Hospital NHS Trist), A Milne (South Tees Hospitals NHS Foundation Trust), H Maclintock (Wrexham Maelor Hospital), C Cleary (Cardiff and the Vale University Health Board), H Murray (NHS Greater Glasgow and Clyde), M Dubia (King's College Hospital NHS Foundation Trust), R Bray 
(North Bristol NHS Trust), R Squire (Plymouth Hospitals NHS Trust), F Hammonds (Royal Cornwall Hospitals NHS Trust).

Contributors DRG is the Chief Investigator of the FASHIoN Trial. DRG led the conception, design, acquisition, analysis and interpretation of data. DRG contributed to drafting and approved the final submitted version of this manuscript. EJD contributed to the design, acquisition, analysis and interpretation of the data. EJD contributed to the drafting and approved the manuscript. PW contributed to the conception, design and acquisition of the trial data. PW contributed to drafting the manuscript and approved the final version. JLD contributed to the conception, design and analysis of trial date. JLD contributed to the drafting and approved the manuscript. NEF contributed to the conception, design, analysis and interpretation of the trial data. NEF contributed to the drafting and approved the manuscript. CEH contributed to the design and analysis of the trial data. CEH contributed to the drafting and approved the manuscript. NP contributed to the conception, design, analysis and interpretation of the data. NP contributed to the drafting and approved the manuscript. SP contributed to the conception, design and interpretation of the data for the trial. SP contributed to the drafting and approved the manuscript. AR contributed to the acquisition, analysis and interpretation of the trial data. AR contributed to the drafting and approved the manuscript. JA contributed to the conception, design and analysis of the trial data. JA contributed to the drafting and approved the manuscript. FA contributed to the analysis and interpretation of the trial data. FA contributed to the drafting and approved the manuscript. AA contributed to the conception and design of the trial. AA contributed to the drafting and approved the manuscript. MLC contributed to the conception and design of the trial. MLC contributed to the drafting and approved the manuscript. JG contributed to the acquisition, analysis and interpretation of trial dat. JG contributed to the drafting and approved the manuscript. $\mathrm{RH}$ contributed to the conception, design and acquisition of the trial data. $\mathrm{RH}$ contributed to the drafting and approved the manuscript. JS contributed to the acquisition, analysis and interpretation of the trial data. JS contributed to the drafting and approved the manuscript.

Funding This work is supported by the Health Technology Assessment Programme of the National Institute of Health Research (Feasibility and Pilot Trial grant number 10/41/02, Full Trial grant number 13/103/02). NF is supported through an NIHR Research Professorship (NIHR-RP-011-015). This project benefited from facilities funded through Birmingham Science City Translational Medicine Clinical Research and Infrastructure Trials Platform, with support from Advantage West Midlands and the Wolfson Foundation. JLD is supported by the MRC ConDuCT Hub for Trials Methodology Research (MR/K025643/1), and the NIHR Collaboration for Leadership in Applied Health Research and Care (CLAHRC) West at University Hospitals Bristol NHS Foundation Trust, and is an NIHR Senior Investigator.

Disclaimer The views expressed are those of the authors and not necessarily those of the NHS, the NIHR or the Department of Health.

Competing interests The chief investigator and many of the co-investigators are orthopaedic surgeons who perform FAl surgery in the NHS and within private practice.

\section{Patient consent Obtained.}

Ethics approval Ethical approval was granted on 12 February 2012 (11/WM0389) and on 1 May 2014 (14/WM/0124), by the Edgbaston Research Ethics Committee.

Provenance and peer review Not commissioned; externally peer reviewed.

Data sharing statement It is our intention to publish the results of the trial in peer-reviewed journals and presented at international meetings. It is not our intention to withhold any data from publication.

Open Access This is an Open Access article distributed in accordance with the terms of the Creative Commons Attribution (CC BY 4.0) license, which permits others to distribute, remix, adapt and build upon this work, for commercial use, provided the original work is properly cited. See: http:// creativecommons.org/licenses/by/4.0/

\section{REFERENCES}

1. Ganz R, Parvizi J, Beck M, et al. Femoroacetabular impingement: a cause for osteoarthritis of the hip. Clin Orthop Relat Res 2003;417:112-20
2. Lavigne M, Parvizi J, Beck M, et al. Anterior femoroacetabular impingement: part I. Techniques of joint preserving surgery. Clin Orthop Relat Res 2004;418:61-6.

3. Ganz R, Leunig M, Leunig-Ganz K, et al. The etiology of osteoarthritis of the hip: an integrated mechanical concept. Clin Orthop Relat Res 2008;466:264-72.

4. Beck M, Kalhor M, Leunig $M$, et al. Hip morphology influences the pattern of damage to the acetabular cartilage femoroacetabular impingement as a cause of early osteoarthritis of the hip. $J$ Bone Joint Surg Br 2005;87:1012-18.

5. Hack K, Di Primio G, Rakhra K, et al. Prevalence of cam-type femoroacetabular impingement morphology in asymptomatic volunteers. J Bone Joint Surg Am 2010;92:2436-44.

6. Gosvig KK, Jacobsen S, Sonne-Holm S, et al. The prevalence of cam-type deformity of the hip joint: a survey of 4151 subjects of the copenhagen osteoarthritis study. Acta Radiol 2008;49:436-41.

7. Montgomery SR, Ngo SS, Hobson T, et al. Trends and demographics in hip arthroscopy in the United States. Arthroscopy 2013;29: $661-5$

8. Botser IB, Smith TW, Nasser R, et al. Open surgical dislocation versus arthroscopy for femoroacetabular impingement: a comparison of clinical outcomes. Arthroscopy 2011;27:270-8.

9. Matsuda DK, Carlisle JC, Arthurs SC, et al. Comparative systematic review of the open dislocation, mini-open, and arthroscopic surgeries for femoroacetabular impingement. Arthroscopy 2011;27:252-69.

10. Excellence NloHaC. Arthroscopic femoro-acetabular surgery for hip impingement syndrome. NICE Guideline (IPG408), 2011.

11. Wall PD, Brown JS, Parsons N, et al. Surgery for treating hip impingement (femoroacetabular impingement). The Cochrane Library, 2014.

12. Emara K, Samir W, Motasem el E, et al. Conservative treatment for mild femoroacetabular impingement. J Orthop Surg (Hong Kong) 2011;19:41-5.

13. Wall PD, Fernandez M, Griffin DR, et al. Nonoperative treatment for femoroacetabular impingement: a systematic review of the literature. PM R 2013;5:418-26.

14. Griffin DR, Wall PD, Realpe A, et al. UK FASHIoN: feasibility study of a randomised controlled trial of arthroscopic surgery for hip impingement compared with best conservative care. Health Technol Assess 2016;20:1-172.

15. Realpe A, Adams A, Wall $P$, et al. A new simple six-step model to promote recruitment to RCTs was developed and successfully implemented. J Clin Epidemiol (in press).

16. Nötzli $\mathrm{H}$, Wyss $\mathrm{T}$, Stoecklin $\mathrm{C}$, et al. The contour of the femoral head-neck junction as a predictor for the risk of anterior impingement. J Bone Joint Surg Br 2002;84:556-60.

17. Nepple JJ, Prather H, Trousdale RT, et al. Diagnostic imaging of femoroacetabular impingement. J Am Acad Orthop Surg 2013;21 (Suppl 1):S20-6.

18. Tönnis $D$, Heinecke A. Acetabular and femoral anteversion: relationship with osteoarthritis of the hip. J Bone Joint Surg Am 1999:81:1747-70.

19. Philippon M, Briggs K, Yen Y-M, et al. Outcomes following hip arthroscopy for femoroacetabular impingement with associated chondrolabral dysfunction minimum two-year follow-up. J Bone Joint Surg Br 2009;91:16-23.

20. Donovan JL, Paramasivan S, de Salis I, et al. Clear obstacles and hidden challenges: understanding recruiter perspectives in six pragmatic randomised controlled trials. Trials 2014;15:5.

21. Mohtadi NG, Griffin DR, Pedersen ME, et al. The development and validation of a self-administered quality-of-life outcome measure for young, active patients with symptomatic hip disease: the International Hip Outcome Tool (iHOT-33). Arthroscopy 2012;28:595-610; quiz 606-10.e1.

22. Walters SJ, Brazier JE. Comparison of the minimally important difference for two health state utility measures: EQ-5D and SF-6D. Qual Life Res 2005;14:1523-32.

23. Oppe M, Devlin NJ, van Hout B, et al. A program of methodological research to arrive at the new international $E Q-5 D-5 L$ valuation protocol. Value Health 2014;17:445-53.

24. Ostendorf $M$, Van Stel $H$, Buskens $E$, et al. Patient-reported outcome in total hip replacement. A comparison of five instruments of health status. J Bone Joint Surg Br 2004;86:801-8.

25. Jenkinson C, Layte R. Development and testing of the UK SF-12. $J$ Health Serv Res Policy 1997;2:14-18.

26. Brazier JE, Roberts J. The estimation of a preference-based measure of health from the SF-12. Med Care 2004;42:851-9.

27. Foster NE, Thomas E, Barlas $P$, et al. Acupuncture as an adjunct to exercise based physiotherapy for osteoarthritis of the knee: randomised controlled trial. BMJ 2007;335:436. 
28. Van den Brink M, Van den Hout WB, Stiggelbout AM, et al. Self-reports of health care utilization: can a questionnaire replace a diary. The 16th annual meeting of the International Society for Technology Assessment in Health Care; The Hague, The Netherlands: ISTAHC, 2000.

29. Amstutz H, Thomas B, Jinnah R, et al. Treatment of primary osteoarthritis of the hip. A comparison of total joint and surface replacement arthroplasty. J Bone Joint Surg Am 1984;66:228-41.
30. National Institute of Health and Care Excellence. Guide to the methods of technology appraisal. NICE Guideline (PMG9), 2013. https://www.nice.org.uk/process/pmg9/chapter/foreword (accessed online Aug 2016).

31. Miles MB, Huberman AM, Saldana J. Qualitative data analysis: a methods sourcebook. London: SAGE Publications, 2013.

32. Ten Have P. Doing conversation analysis. London: Sage, 2007. 